\title{
A boronate gel-based synthetic platform for closed-loop insulin delivery systems
}

\author{
Akira Matsumoto $\mathbb{1}^{1,2} \cdot$ Siyuan Chen $^{3}$ \\ Received: 20 April 2021 / Revised: 20 May 2021 / Accepted: 20 May 2021 / Published online: 20 July 2021 \\ (c) The Author(s) 2021. This article is published with open access
}

\begin{abstract}
Diabetes is one of the most devastating global diseases with an ever-increasing number of patients. Achieving persistent glycemic control in a painless and convenient way is an unmet goal for diabetes management. Insulin therapy is commonly utilized for diabetes treatment and usually relies on patient self-injection. This not only impairs a patient's quality of life and fails to precisely control the blood glucose level but also brings the risk of life-threatening hypoglycemia. "closed-loop" insulin delivery systems could avoid these issues by providing on-demand insulin delivery. However, safety concerns limit the application of currently developed electronics-derived or enzyme-based systems. Phenylboronic acid (PBA), with the ability to reversibly bind glucose and a chemically tailored binding specificity, has attracted substantial attention in recent years. This focus review provides an overview of PBA-based versatile insulin delivery platforms developed in our group, including new PBA derivatives, glucose-responsive gels, and gel-combined medical devices, with a unique "skin layer" controlled diffusion feature.
\end{abstract}

\section{Introduction}

As the seventh leading cause of death, diabetes mellitus is one of the most challenging global issues [1,2]. More than 463 million people were living with diabetes in 2019 , and the number is expected to increase dramatically to 700 million in 2045 [3]. Insulin is commonly used for the treatment of patients with type I and advanced type II diabetes and is usually self-administered by subcutaneous injection multiple times per day [4-6]. It is difficult to maintain persistent glycemic control, and more importantly,

Akira Matsumoto

matsumoto.bsr@tmd.ac.jp

Siyuan Chen

siyuan.chen@njtech.edu.cn

1 Institute of Biomaterials and Bioengineering, Tokyo Medical and Dental University, Tokyo, Japan

2 Kanagawa Institute of Industrial Science and Technology, Ebina, Japan

3 Research Institute for Biomaterials, Tech Institute for Advanced Materials, College of Materials Science and Engineering, Suqian Advanced Materials Industry Technology Innovation Center, NJTech-BARTY Joint Research Center for Innovative Medical Technology, Nanjing Tech University, Nanjing, China hypoglycemia caused by overdose may lead to behavioral and cognitive disturbance, brain damage, or even death [7, 8]. Therefore, "closed-loop" insulin delivery systems mimicking the pancreas to secrete insulin in response to hyperglycemia are highly desirable [9-11]. Electronicsbased artificial pancreas can efficiently control blood glucose levels and reduce the risk of hypoglycemia $[9,12,13]$. However, its high cost, risk of electronic failures, discomfort associated with needle-tipped catheter insertion under the skin, a long time to establish a learning curve, and frequent calibration limit its wide application $[14,15]$.

Consequently, electronics-free, chemically controlled glucose-responsive systems have attracted constant research interest [16-18]. Compared to the widely exploited glucosesensitive enzymes such as glucose oxidase $\left(\mathrm{GO}_{x}\right)$ and glucosebinding proteins such as concanavalin $\mathrm{A}$ (Con $\mathrm{A})$, synthetic phenylboronic acid (PBA) has advantages due to its high stability, durable glucose sensitivity, suitability for large-scale production, and lack of denaturation and immunotoxicity concerns [19-22]. It can reversibly bind with 1,2- or 1,3-cisdiols, including glucose, through boronate ester formation in an aqueous solution [23, 24]. By fine-tuning the stereochemistry and electronic affinity, the binding strength and specificity of PBA with glucose can be well controlled [25-27]. All these characteristics make PBA an attractive candidate for glucose sensing and self-regulated insulin delivery. 


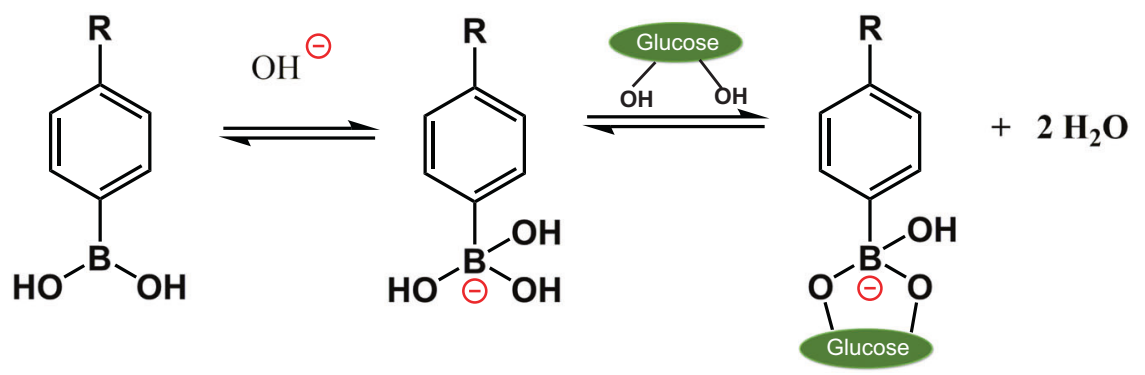

Scheme 1 Glucose-dependent equilibria of PBA derivatives

A<smiles>OB(O)c1ccccc1</smiles>

PBA<smiles>[B]=CC(=O)NCCNC(=O)c1ccc(B(O)O)cc1</smiles>

DDOPBA
C<smiles>C=CC(=O)Nc1cccc(B(O)O)c1</smiles>

AAPBA<smiles>O=CC(=O)NCCNC(=O)c1ccc(B(O)O)cc1F</smiles>

$A_{m}$ ECFPBA
E

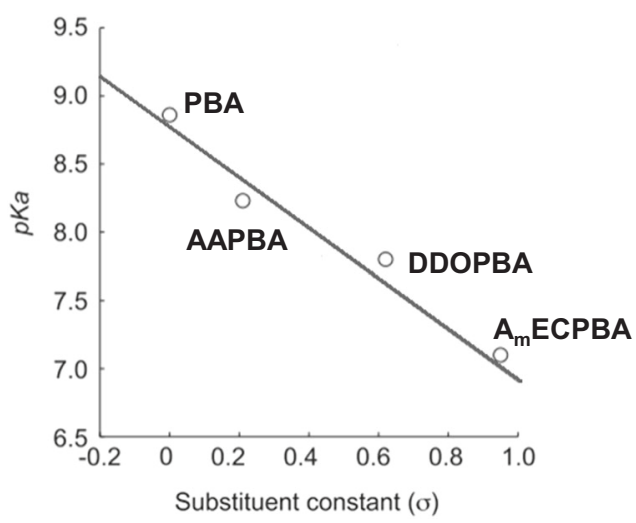

Fig. 1 Chemical structure of $\mathbf{A}$ PBA and $\mathbf{B}-\mathbf{D}$ its derivatives. $\mathbf{E}$ The linear relationship between $p K_{a}$ values and substituent constants of various PBAs

In this focus review, we summarized the recent progress our group achieved in the area of PBA-based glucose-responsive systems for self-regulated insulin delivery, including PBA monomers with decreased $p K_{a}$ values, PBA-based glucose-responsive gels, and PBA gelcombined catheter and hollow fiber medical devices. Their therapeutic effectiveness was validated in vivo. In addition, PBA hydrogels containing microneedles (MNs) for noninvasive transdermal application will be described.

\section{PBA monomers with decreased $p K_{a}$ values}

As a Lewis acid, PBA in aqueous solution exists in equilibrium between a neutrally charged trigonal planar state and an anionic tetrahedral form in dynamic balance $[28,29]$. When the $\mathrm{pH}$ of the solution is below the $p K_{a}$ of PBA, most of the moieties are in neutral form and relatively hydrophobic. When the $\mathrm{pH}$ value is above the $p K_{a}$ of PBA, the majority of the moieties are negatively charged. Upon the addition of glucose, the anionic tetrahedral form can covalently bind with glucose in a reversible way, shifting the equilibrium toward the anionic form of PBA (Scheme 1) [30-32]. As a result, it is commonly observed that the apparent $p K_{a}$ of PBA decreases with the addition of glucose [33]. Such a glucose-dependent change in the PBA equilibria, when taking place in a gel network, translates into a change in the counterions' osmotic pressure, which dramatically affects the state of hydration of the gel. This dynamic PBA-glucose interaction gives PBA-functionalized materials great potential in glucose-responsive drug delivery [23, 34]. However, at physiological $\mathrm{pH}$, charged phenylboronate, which is responsible for the complex with glucose, is formed in a limited fraction due to the relatively high $p K_{a}$ of PBA (typically around 9, Fig. 1A) [35]. Thus, the $p K_{a}$ of PBA needs to be decreased to achieve adequate function under physiological $\mathrm{pH}$ $[36,37]$.

In our group, a variety of PBA derivatives containing electron-withdrawing substituents on the phenyl ring were reported. By the introduction of a para-positioned carbamoyl group into the phenyl ring, 4-(1,6-dioxo-2,5-diaza-7oxamyl) phenylboronic acid (DDOPBA, Fig. 1B) was 
A

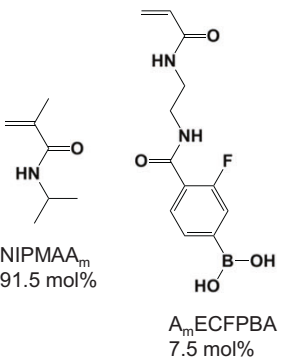

B

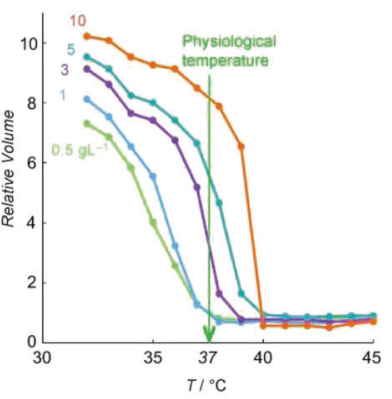

C

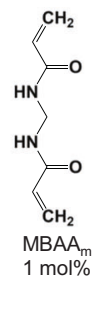

D

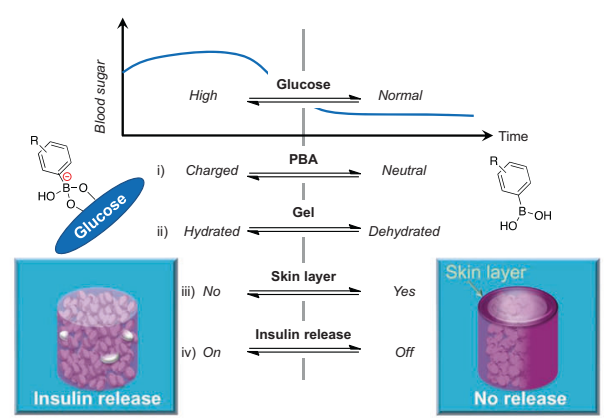

E

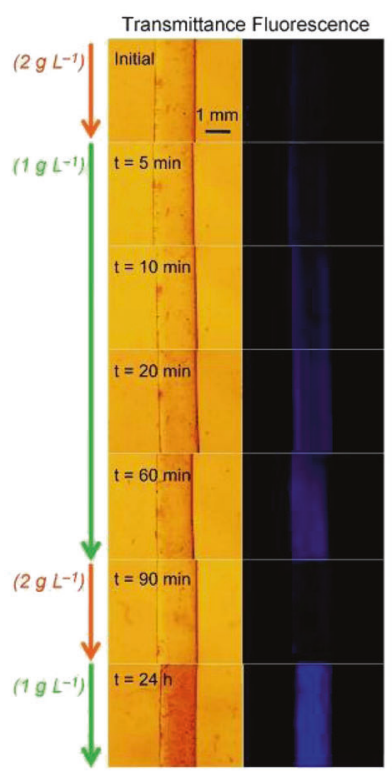

$\mathbf{F}$

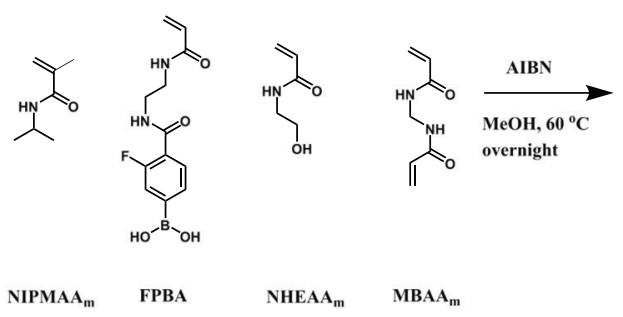

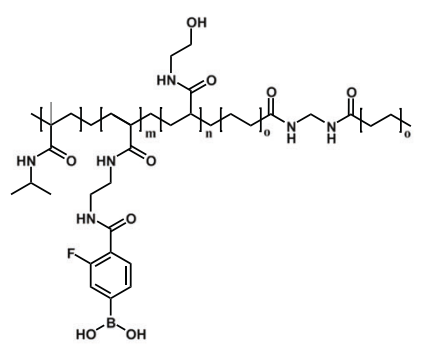

G

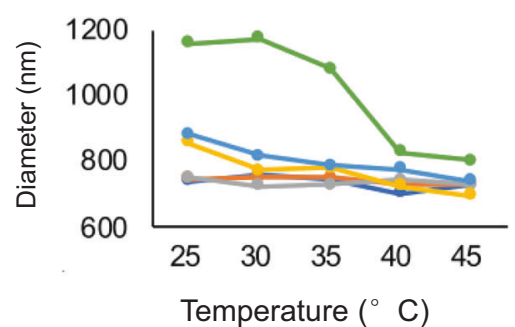

Fig. 2 A Optimized monomers and their molar amounts in the gel solution to achieve glucose responsiveness under physiological conditions. B Phase diagram of the gel showing the equilibrium volume changes under various temperatures and glucose concentrations at $\mathrm{pH}$ 7.4. C Time-course changes in the fluorescence intensity of FITCinsulin released from the gel under physiological conditions and the temporal patterns of the fluctuation in glucose concentration.

synthesized [33, 38, 39]. Compared to its counterpart 3acrylamidophenylboronic acid (AAPBA, $p K_{a}=8.2$, Fig. 1C) with a meta-positioned amide group [23], DDOPBA shows a reduced $p K_{a}$ value of 7.8 due to the relatively larger substituent constant $(\sigma=0.62)$ of the parapositioned carbamoyl group and its strong electronwithdrawing effect. To further decrease the $p K_{a}$, a metafluoro group with a strong electron affinity to the phenyl ring was introduced to DDOPBA [40]. Usually, a fluorocarbon group is very hydrophobic, which may reduce the phase transition temperature of the PBA-bearing gel and limit its application [41]. However, the new derivative 4(2-acrylamidoethylcarbamoyl)-3-fluorophenylboronic acid $\left(\mathrm{A}_{\mathrm{m}}\right.$ ECFPBA, Fig. 1D) containing para-carbamoyl and meta-fluoro substituents is capable of decreasing this side effect. The $p K_{a}$ value is decreased to 7.2 , which is ideally lower than the physiological $\mathrm{pH}$.
D Schematic of "skin layer" controlled self-regulated insulin release. E Time-course transmittance and fluorescence images of a cylindershaped gel under different glucose concentrations. F Chemical structure of the gel with a temperature-independent function. G Phase diagrams showing the diameter change of the gel with optimized formulation at various glucose concentrations. Reproduced with permission from refs. [40, 45, 46]

\section{PBA-based glucose-responsive gels}

To take one step further, $A_{m}$ ECFPBA was combined with acrylamide and methacrylamide to create copolymer gels for on-demand insulin delivery [40, 42]. By fine-tuning the hydrophobic/hydrophilic balance, the synthesized gel with an optimized formulation (NIPMAA ${ }_{m}: A_{m}$ ECFPBA: $_{\text {MBAA }}=$ 91.5:7.5:1, Fig. 2A) displayed a volume phase transition corresponding to the glucose concentration, with a threshold value of normal glucose level $(100 \mathrm{mg} / \mathrm{dL})$ at physiological

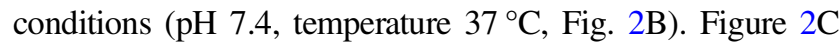
shows the time-course insulin release profile from the gel under different glucose treatment patterns under physiological conditions. The release of insulin from the PBA gel was well correlated with the glucose pattern change. Furthermore, when increasing the glucose concentration from $1-2$ to $3 \mathrm{~g} / \mathrm{L}$, a tenfold increase in the insulin release rate was observed. 
Under normoglycemia conditions for at least $16 \mathrm{~h}$, no insulin release was detected. These results demonstrate that this protein-free, totally synthetic PBA gel can function as a selfregulated insulin delivery system that is capable of tightly regulating the released insulin dosage without the risk of causing hypoglycemia.

The mechanism of self-regulated insulin release was further explored. As explained in Fig. 2D, due to the glucose-dependent shift in the equilibria of PBA, upon an abrupt decrease in glucose concentration, the surface of a PBA-containing gel changes rapidly from a swollen hydrophilic state into a shrunken hydrophobic state. The thus-formed "skin layer," which is a microscopically thin, dehydrated layer [43], was able to control the diffusion of the loaded insulin molecules. Since the binding of PBA with glucose is reversible, if the glucose concentration rises again, the skin layer disappears with gel hydration, and the amount of insulin released is restored. Hence, by optimizing the chemical structure of PBA (tuning the $p K_{a}$ ), the crosslinking density, and the molar ratio of various monomers, the skin layer could rapidly form at normal blood glucose levels, thus effectively switching insulin release in a "gated" manner when hitting the normoglycemia level under physiologically relevant conditions [26, 42, 44].

The formation of the expected skin layer was confirmed by confocal microscopy with 8-anilino-1-naphthalene sulfonic acid (ANS) as an environmentally responsive pigment. Since the fluorescence intensity of ANS is increased when the permittivity of its surrounding environment decreases, the formation of the skin layer, which leads to reduced water content on the gel surface, could be visualized by this method [40]. As shown in Fig. 2E, under hyperglycemic conditions $(2 \mathrm{~g} / \mathrm{L})$, negligible fluorescence intensity was observed due to hydration of the gel. Instead, a dramatic increase in fluorescence intensity is observed when decreasing the glucose concentration to the normal blood sugar level $(1 \mathrm{~g} / \mathrm{L})$, indicating the formation of the skin layer. When returning to the hyperglycemic state, the fluorescence suddenly disappeared, confirming the reversible formation of the skin layer. Thus, this gel system could effectively switch insulin release in a "gated" manner after reaching the normoglycemia level of glucose.

To further eliminate the safety concern that hampers the clinical application of the gel, its temperature dependency should be minimized. One straightforward approach is to decrease the thermosensitive NIPMAA $A_{m}$ fraction. However, this consequently increases the $\mathrm{A}_{\mathrm{m}}$ ECFPBA fraction and thus leads to excessively enhanced hydrophobicity and results in loss of function under physiological temperature [40]. To solve this dilemma, hydrophilic $N$-hydoroxyethylacrylamide $\left(\mathrm{NHEAA}_{\mathrm{m}}\right)$-bearing hydroxyl groups were introduced in the gel network to serve as intermolecular cross-linkers via boronate ester formation with
$A_{m}$ ECFPBA while also increasing the hydrophilicity of the overall network (Fig. 2F). By fine-tuning the hydrophobic/ hydrophilic balance, the optimum formulation (NIPMAA m $_{\text {: }}$ FPBA:NHEAA $\left.{ }_{\mathrm{m}}: \mathrm{MBAA}_{\mathrm{m}}=39.56: 16.95: 38.48: 5.00\right)$ achieved a temperature-independent closed-loop function in the temperature range of $25-45^{\circ} \mathrm{C}$ (Fig. $2 \mathrm{G}$ ), which is critical for in vivo application to avoid unwanted burst insulin release immediately after implantation.

\section{PBA gel-combined devices}

Our totally synthetic gel-based technology, taking advantage of the skin-layer mechanism, should offer crucial advantages over those involving proteins or nanoparticle formulations in terms of establishing both acute- and longterm release controls. In addition, the readiness of the preparation enables a combination with existing medical devices. Based on this idea, we developed a cathetercombined device [45]. Figure 3A shows the morphology and preparation method of this catheter-combined device. The pregel solution was loaded into a 4-French silicone catheter bearing penetrating openings to fill the whole inner wall and the openings, followed by gelation upon heating. Glucose-dependent insulin release could be well controlled by the skin layer formed in the openings (Fig. 3B). To increase the biocompatibility and reinforce the mechanical integrity, the surface of the device was coated with poly (ethylene glycol). The gel-modified catheter was then cut into $15-\mathrm{mm}$ long pieces and connected with another nontreated long catheter $(11 \mathrm{~cm})$ serving as an insulin reservoir. This catheter device meets the clinical biosafety requirements according to in vitro cytotoxicity and histological examinations (Fig. 3C).

The therapeutic effectiveness of the catheter device was evaluated in vivo. In the glucose tolerance test (Fig. 3D), the glucose-lowering effect was observed as soon as $15 \mathrm{~min}$ after glucose injection in healthy mice implanted with a gelcombined catheter. In line with this, a remarkable increase in serum insulin was detected synchronized with acute glucose injection. More advantageously, therapeutic effectiveness was well maintained even after 3 weeks of implantation. These results clearly demonstrate the durability and stability of this device. The glucose-responsive insulin release of this device was further examined in diabetic mice. A significant decrease in blood glucose concentration was noted in diet-induced type II diabetic mice treated with the recombinant human insulinloaded catheter device (Fig. 3E). More importantly, the device treatment remarkably decreased the serum mouse C-peptide concentration (Fig. 3F), indicating that the device effectively mitigates obesity-induced hyperinsulinemia. Figure 3G shows the glucose tolerance test in STZ-induced type I diabetic mice. The catheter device effectively decreased the blood glucose 
A

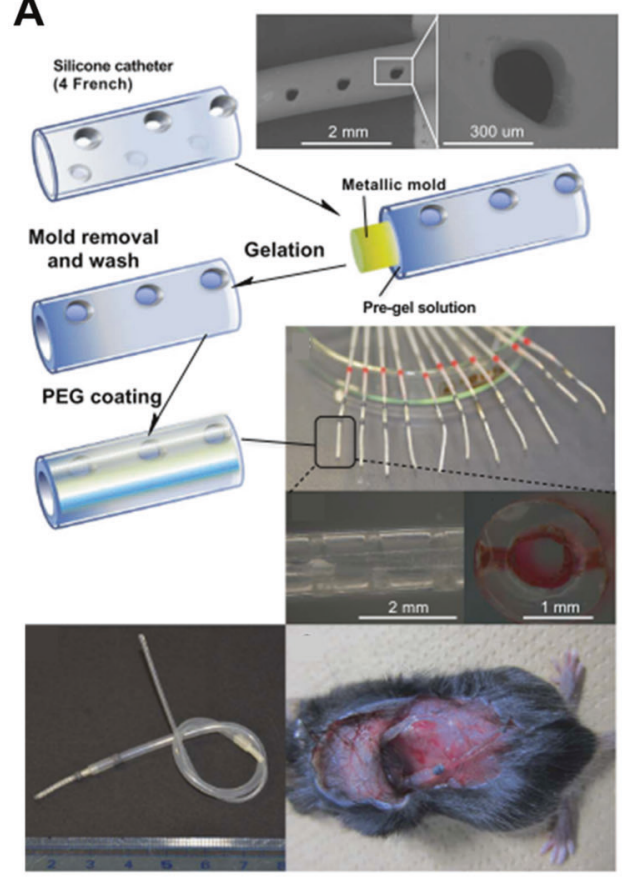

B

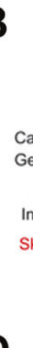

D
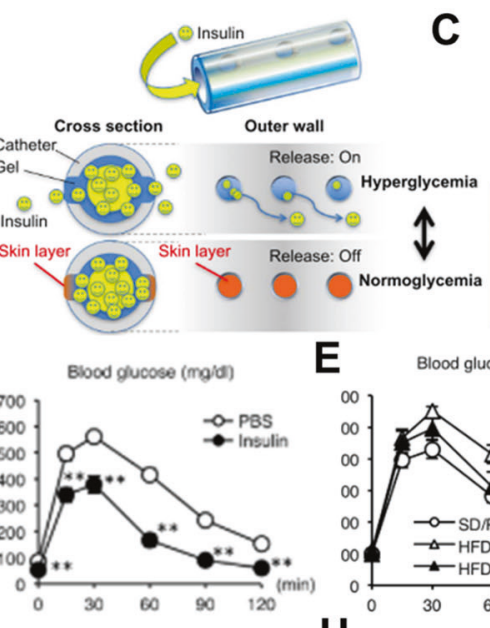

G
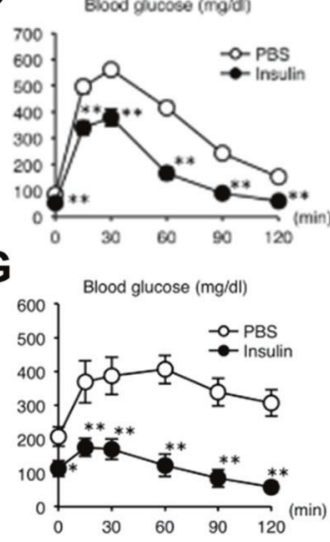
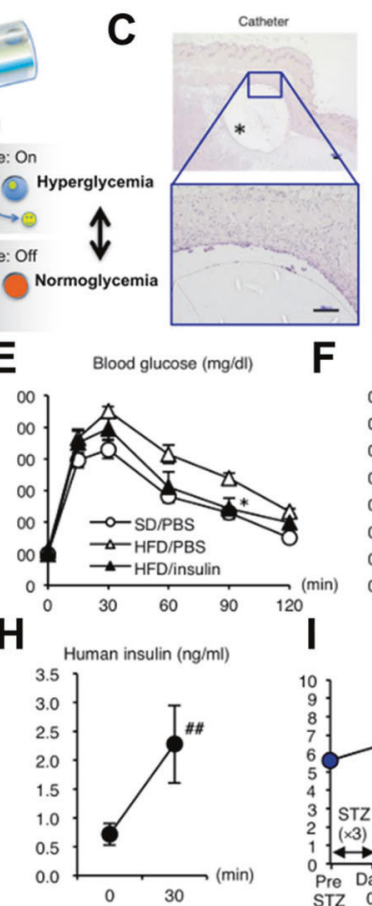

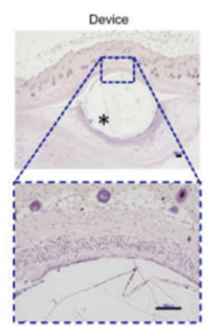

Mouse C-peptide (ng/ml)
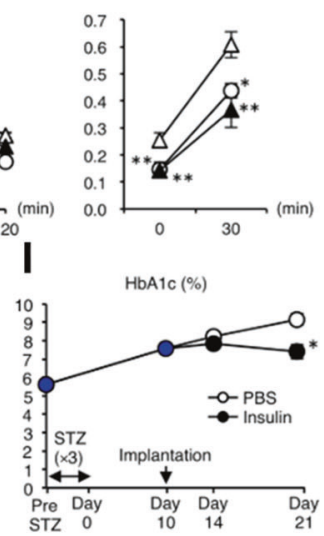

Fig. 3 A Morphology and fabrication method of the catheter-combined device. B Schematic of glucose-responsive insulin regulated by the skin layer. C H\&E staining images showing the subcutaneous tissue surrounded by the implanted catheters. D Glucose tolerance test (GTT) of healthy mice implanted with catheter devices containing PBS and human insulin. E Blood glucose concentration and $\mathbf{F}$ mouse C-peptide concentration of type II diabetic mice in the GTT. G Blood glucose concentration and $\mathbf{H}$ human serum insulin concentration of type I diabetic mice in the GTT. I HbA1c levels of type I diabetic mice implanted with devices. Reproduced with permission from ref. [45] concentration to a normal level within $1 \mathrm{~h}$ and effectively controlled glucose fluctuation. Considerably increased human insulin was detected in the serum (Fig. 3H), and a significantly decreased hemoglobin Alc (HbAlc) level was observed in type I diabetic mice treated with the insulin-containing device after 21 days (Fig. 3I). This smart catheter-combined device could show therapeutic effectiveness under both insulindeficient and insulin-resistant conditions, suggesting its potential for clinical application.

To further explore the scalability and efficacy for daily blood glucose fluctuations, we recently developed a hollow fiber-combined device that is scaled suitably for rats [46]. Due to the diffusion-dependent nature of the gel, the insulin release rate could be easily scaled up by increasing the surface area of the gel. Hence, we introduce a thin coating of the gel with temperature-independent behavior throughout the hemodialysis fiber to develop a hollow fiber device (Fig. 4A). Compared to the previously described catheter-combined device, this hollow fiber device consists of 25 fibers and thus dramatically increases the diffusion-active surface area. The gel-installed region was protected by a cellulose dialysis membrane to prevent fibrillization during implantation and connected with an insulin reservoir by glue (Fig. 4B). In vitro insulin release challenged by different glucose patterns was investigated and further analyzed by a mathematical model to gain a quantitative basis for scaling. In vivo evaluation in type I diabetic rats was performed, and the weight was approximately ten times that of the mice. The in vivo results suggest that the subcutaneously implanted device responded rapidly to acute blood glucose level changes and that the blood glucose-lowering effect was sustainable for at least 7 days (Fig. 4C). Figure 4D shows remarkably decreased HbAlc levels in the diabetic rats treated with the insulinloaded device. No significant change in the population of white blood cells was observed, suggesting its biosafety. Remarkably, this device could even show a marked benefit in coping with glucose spike-like symptoms (daily glucose fluctuations) over the timescale of a day, to our knowledge, for the first time using an electronics-free system (Fig. 4E). Recent clinical studies have revealed that not only sustained elevation of blood glucose levels but also glucose fluctuation represents key determinants for diabetic complications and mortality, thus forming the consensus that care must be taken to evaluate the daily blood glucose fluctuations as much as the commonly used fasted blood glucose levels and HbA1c to maximize the efficacy of the treatment. With inherent stability, diffusion-dependent scalability, and week-long and acute glucose responsiveness, our PBA gel technology may offer a low-cost alternative to current electronics-based approaches. 


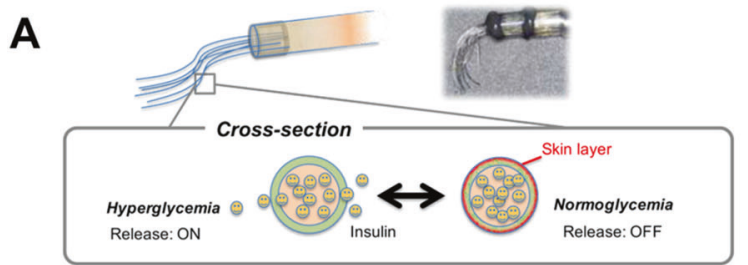

B
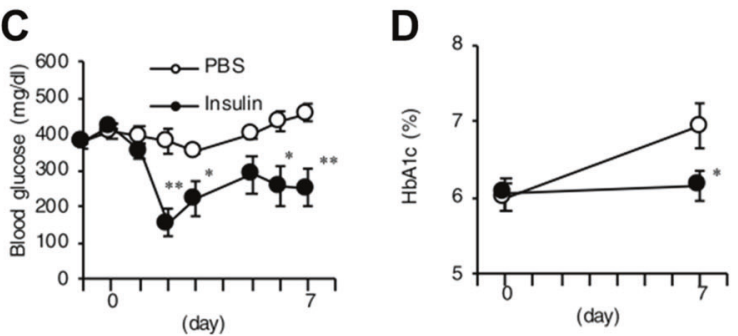

Fig. 4 A Schematic of the gel-combined hollow fiber with closed-loop functionality. B The appearance of the hollow fiber device. C The blood glucose concentration of type I rats implanted with hollow fiber devices containing PBS or human insulin. D HbA1c levels of type I

\section{PBA gel containing MNs}

Traditional insulin injection is painful and inconvenient and may be associated with microbial contamination and nerve damage [5, 6]. MN technology has the possibility to overcome these limitations. MNs typically contain sharper needles than hypodermic needles, with a length range of approximately several hundred micrometers $[47,48]$. These characteristics enable MNs to effectively disrupt the stratum corneum, which is the main barrier that hampers the transdermal delivery of insulin [49-51]. Since MNs only form microscale channels on the skin and the needle length is not long enough to reach the nerve endings in the dermis, an MN patch is minimally invasive and could eliminate injection pain [52-54]. By incorporating glucose-sensitive elements into MNs, it is promising to achieve on-demand painless insulin delivery without human intervention [55-57].

Our recent work reported an enzyme-free MN array patch composed of a PBA-containing hydrogel that established sustained and acute glucose-responsive insulin delivery (Fig. 5A) [58]. To ensure sufficient mechanical strength for skin insertion, biocompatible and mechanically robust silk fibroin derived from the silkworm Bombyx mori was incorporated into the NIPAA $A_{m} / A_{m}$ ECFPBA hydrogel to form a semi-interpenetrating network (Fig. 5B). As shown in Fig. $5 \mathrm{C}$, an increased wall thickness was observed in the cross-sectional morphology of the silk fibroin-containing semi-interpenetrating network hydrogel. Methanol treatment to physically crosslink the silk fibroin via intermolecular $\beta$ sheet crystallization further enhanced the surface roughness, indicating improved mechanical strength. To fabricate MNs utilizing this hybrid hydrogel, six fabrication methods were investigated and compared [59]. The two-layer strategy,
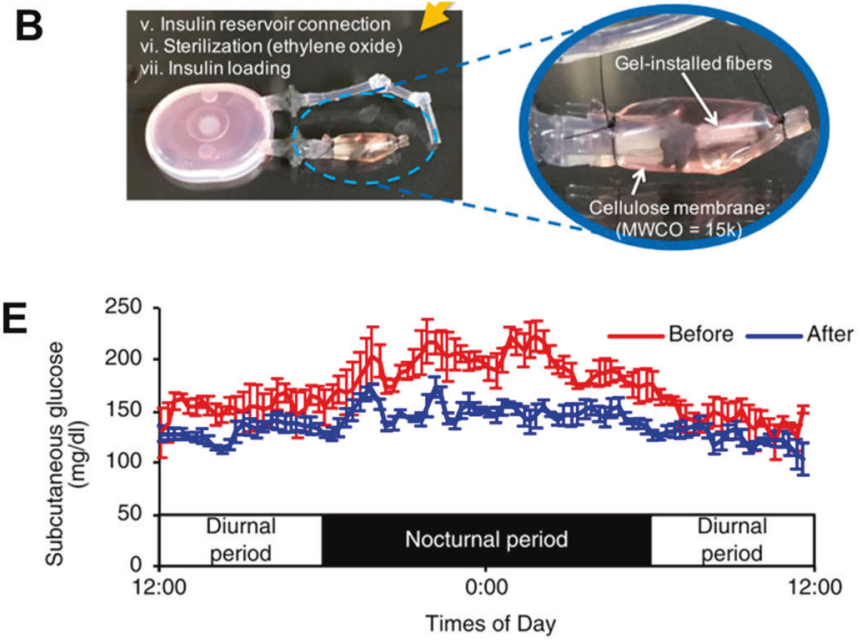

rats before and after 7 days of implantation. E Daily glucose fluctuations in mildly diabetic rats before and after device implantation. Reproduced with permission from ref. [46]

which allows the needle region to be formed by the semiinterpenetrating network hydrogel and the base layer part to be fabricated by silk fibroin, was chosen for MN fabrication, as it successfully formed sharp needle tips and avoided deformation of the base layer (Fig. 5D). Figure 5E shows the uniform distribution of insulin and hydrogel throughout the needle region. Due to the incorporation of mechanically robust silk fibroin, the MN array patch displayed considerably stronger mechanical strength than the other polymeric MNs and was capable of effectively penetrating the skin to create micropores for transdermal insulin delivery (Fig. 5F). The $\mathrm{MN}$ array patch was highly stable in an aqueous environment, showing no deformation and negligible weight loss even after 2 months of incubation in pH $7.4 \mathrm{PBS}$ at $37^{\circ} \mathrm{C}$ (Fig. 5G). More advantageously, as seen in Fig. 5H, acute and long-term insulin release in a manner that is highly synchronized with the glucose pattern was observed in this $\mathrm{MN}$ patch. Weekly long glucose-responsive insulin release could be achieved by reloading insulin in the drug reservoir, indicating its potential to provide long-term glycemic control without human intervention.

However, safety concerns remain because gel behavior is highly dependent on temperature, and skin temperature is remarkably affected by a number of factors, such as exercise, illness, and environmental changes. To solve this problem, the abovementioned temperature-independent formulation was applied to the MN patch [60], where the content of NHEAA $\mathrm{m}_{\mathrm{m}}$ was carefully optimized (Fig. 6A). As demonstrated in the phase diagram (Fig. 6B), with the optimized hydrogel formulation, that is, $60.7-\mathrm{mol}^{2} \quad \mathrm{NIPAA}_{\mathrm{m}}, \quad 10.7-\mathrm{mol} \%$ $\mathrm{A}_{\mathrm{m}}$ ECFPBA, and 23.8-mol\% NHEAA $\mathrm{m}_{\mathrm{m}}$, the glucosedependent change in hydration was established in a temperature-insensitive fashion. A porous structure was 
A

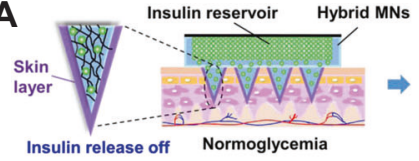

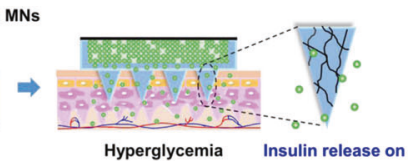

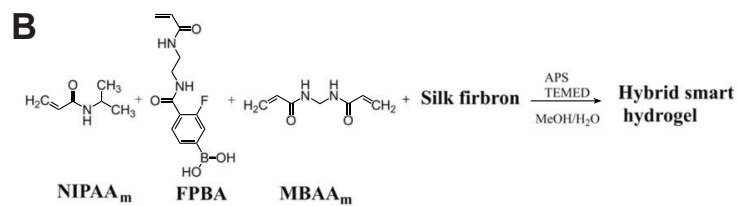

C
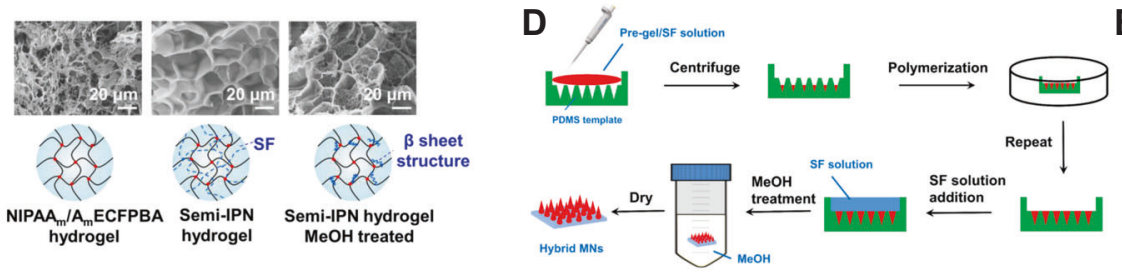

E

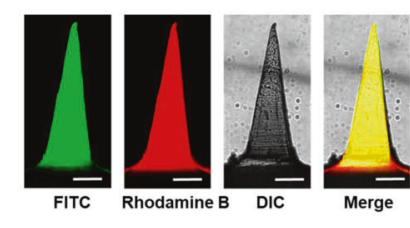

$\mathbf{F}$

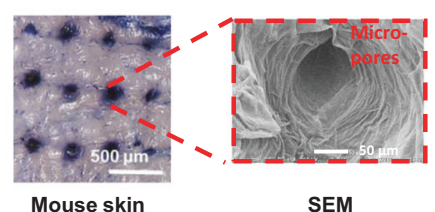

G

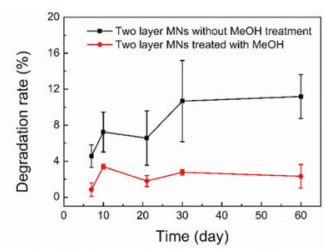

H
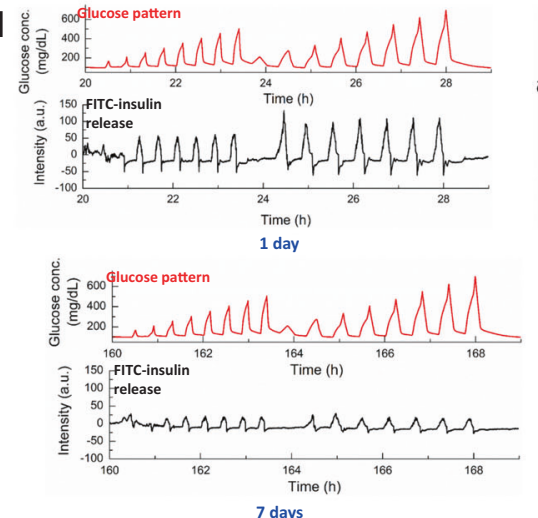
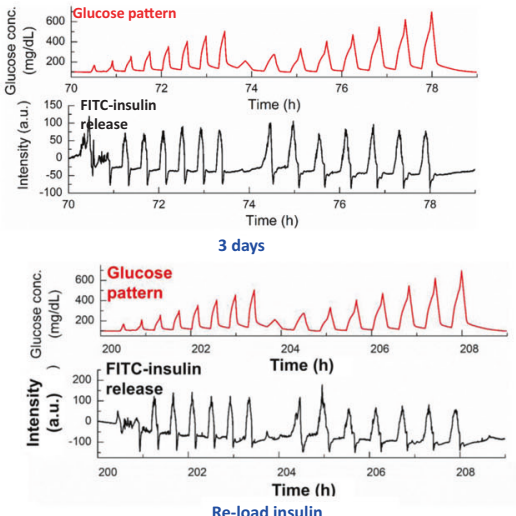

Fig. 5 A Schematic of a glucose-responsive gel combined with an MN array patch. B Synthesis of silk fibroin-composed semi-interpenetrating network hydrogel. C Cross-sectional SEM images of hydrogels. D Schematic of MN fabrication using a two-layer method. E Distribution of FITC-insulin and rhodamine B-traced hydrogels in the MN

observed in this gel, indicating suitability for drug delivery (Fig. 6C). The gel was fabricated into a totally synthetic MN array patch using a two-layer strategy as described above, using crystallized PVA as the base layer (Fig. 6D, E). Rapid insulin release control of the two-layer MNs to the programmed glucose pattern was observed over a wide range of temperatures $\left(28-39^{\circ} \mathrm{C}\right.$, Fig. $\left.6 \mathrm{~F}\right)$, which could successfully eliminate the safety concerns of inadequate insulin release due to skin temperature changes. Due to the skin-layer driven diffusion mode, this temperature-insensitive $\mathrm{MN}$ patch could also provide glucose-responsive insulin release for several days, which shows advantages compared to the other glucose-sensitive $\mathrm{MN}$ patches that relay on $\mathrm{GO}_{x}$. Insulin pharmacokinetics could be further tailored by chemical modification of the PBA and hydrogel, thus showing promise for personalized treatment.

\section{Conclusions}

This focus review describes the up-to-dateprogress of PBAbased versatile delivery platforms developed in our group array patch. F Micropores formed on the mouse skin after MN treatments. G Long-term stability assay of the $\mathrm{MN}$ array patch. $\mathbf{H}$ In vitro glucose-responsive insulin release from the MN array patch on days 1, 3 , and 7 and after insulin reloading. Reproduced with permission from ref. [58]

for on-demand insulin delivery. Due to the inherent advantages of PBA, our smart gel technology could provide diabetic patients with long-lasting, rapid on-demand insulin delivery as an alternative to traditional methods. By combining the smart gel with well-established medical devices such as catheters and MNs, convenient and effective insulin treatment could be achieved in a user-friendly, less invasive, and inexpensive way, which not only benefits patients in developing countries that have limited access to healthcare facilities but also less motivated patients such as the elderly, infants, and people who have trypanophobia.

Chemically controlled glucose-responsive insulin systems that are based on GOx, Con A, and PBA possess great advantages compared to manual subcutaneous injection. However, to transition from research to clinical practice, several challenges need to be addressed, including the precise control of insulin release behavior, large-scale production and quality control, and their biosafety and clinical effectiveness. Our continuous efforts will be devoted to optimizing the gel's release control ability, simplifying the fabrication methods to realize 


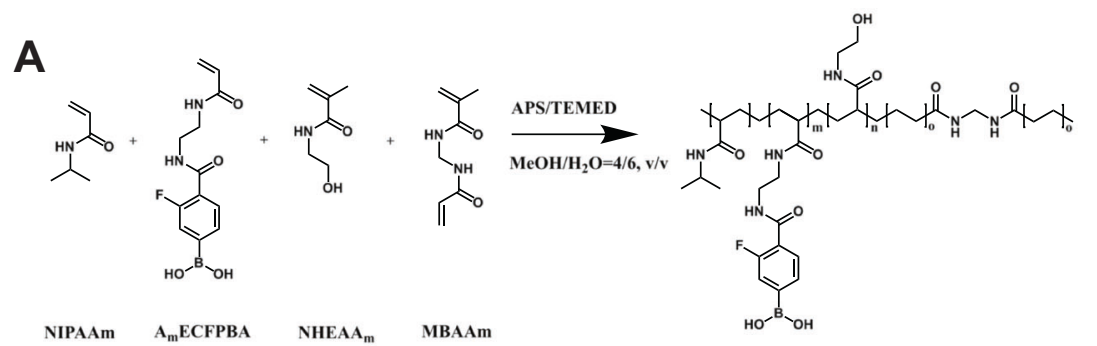

$\mathbf{F}$
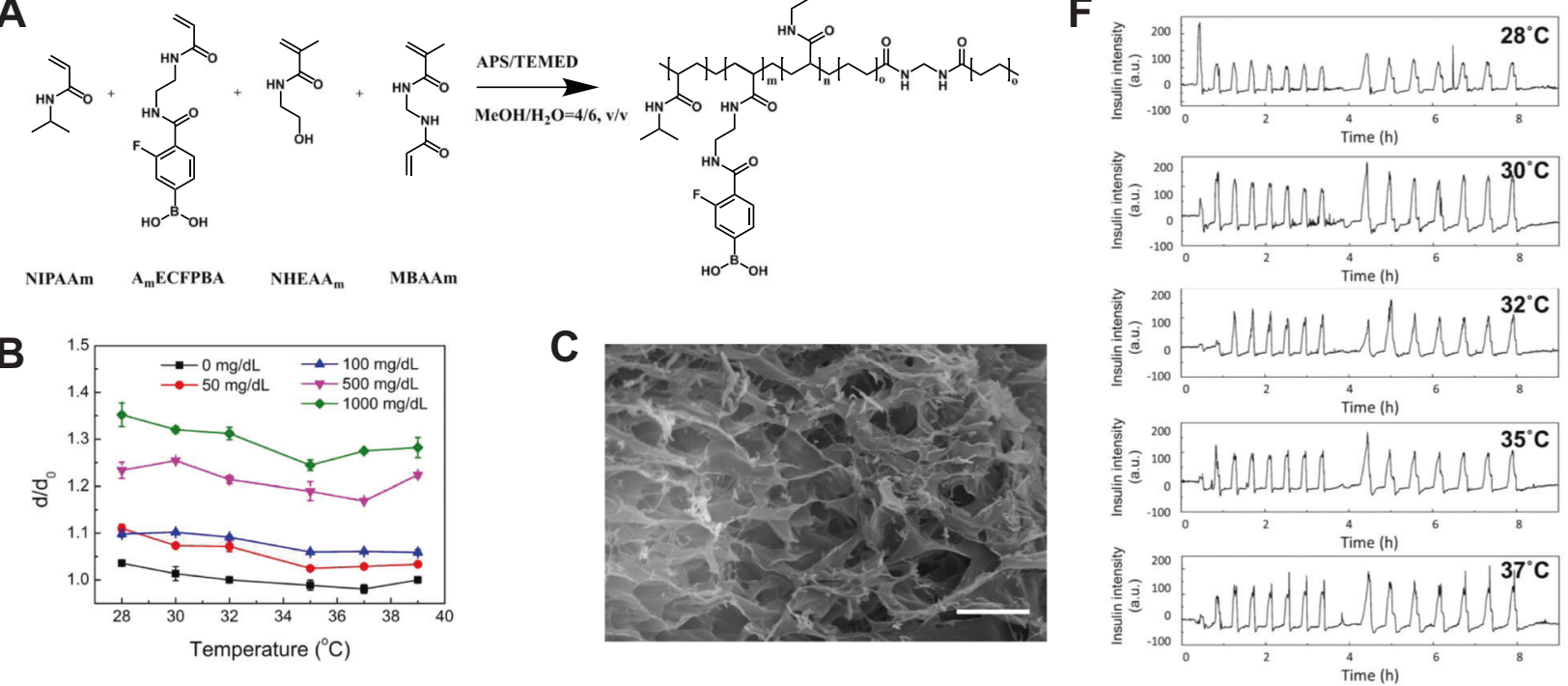

D

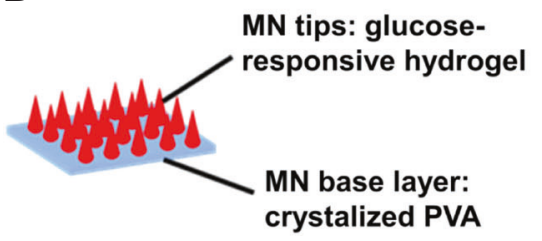

E

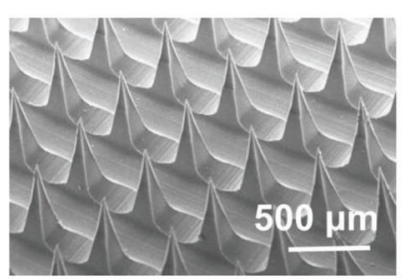

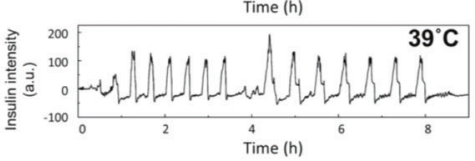

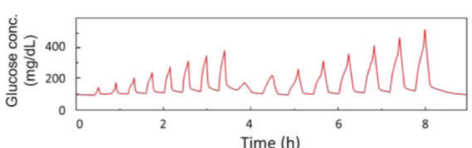

Fig. 6 A Chemical structure of the temperature-stable boronate gel. B Phase diagrams showing the gel diameter change under various glucose concentrations and temperatures. C Cross-sectional SEM images of the gel. D Schematic representation and morphology of the totally synthetic $\mathrm{MN}$ array patch. $\mathbf{F}$ In vitro insulin release from the $\mathrm{MN}$ array patch at various temperatures and the temporal pattern of the fluctuation in glucose concentration. Reproduced with permission from ref. [60] industrial mass production, and validating its effectiveness in clinical trials. By addressing these issues, our smart gel-based technology will have great potential to impact future diabetes treatment.

Acknowledgements This work was supported in part by grants-in-aid for scientific research from the Ministry of Education, Culture, Sports, Science and Technology of Japan (MEXT), the Cooperative Research Project of Research Center for Biomedical Engineering (MEXT), Program for Building Regional Innovation Ecosystem (MEXT), and Japan Science and Technology Agency (JST) COI Grant Number JPMJCE1305.

\section{Compliance with ethical standards}

Competing interests The authors declare no competing interests.

Publisher's note Springer Nature remains neutral with regard to jurisdictional claims in published maps and institutional affiliations.

Open Access This article is licensed under a Creative Commons Attribution 4.0 International License, which permits use, sharing, adaptation, distribution and reproduction in any medium or format, as long as you give appropriate credit to the original author(s) and the source, provide a link to the Creative Commons license, and indicate if changes were made. The images or other third party material in this article are included in the article's Creative Commons license, unless indicated otherwise in a credit line to the material. If material is not included in the article's Creative Commons license and your intended use is not permitted by statutory regulation or exceeds the permitted use, you will need to obtain permission directly from the copyright holder. To view a copy of this license, visit http://creativecommons. org/licenses/by/4.0/.

\section{References}

1. Zimmet P, Alberti KG, Magliano DJ, Bennett PH. Diabetes mellitus statistics on prevalence and mortality: facts and fallacies. Nat Rev Endocrinol. 2016;12:616-22.

2. W.H. Organization. Global report on diabetes. Geneva; 2016.

3. Saeedi P, Petersohn I, Salpea P, Malanda, B, Karuranga S, Unwin $\mathrm{N}$, et al. Global and regional diabetes prevalence estimates for 2019 and projections for 2030 and 2045: results from the International Diabetes Federation Diabetes Atlas, 9th edition. Diabetes Res Clin Pract. 2019;157:107843.

4. Zaykov AN, Mayer JP, DiMarchi RD. Pursuit of a perfect insulin. Nat Rev Drug Discov. 2016;15:425-39.

5. Zhang Y, Yu J, Kahkoska AR, Wang J, Buse JB, Gu Z. Advances in transdermal insulin delivery. Adv Drug Deliv Rev. 2019;139:51-70

6. Pillai O, Panchagnula R. Insulin therapies-past, present and future. Drug Discov Today. 2001;6:1056-61. 
7. Owens DR, Zinman B, Bolli GB. Insulins today and beyond. Lancet. 2001;358:739-46.

8. Pickup JC. Insulin-pump therapy for type 1 diabetes mellitus. $\mathrm{N}$ Engl J Med. 2012;366:1616-24.

9. Hovorka R. Closed-loop insulin delivery: from bench to clinical practice. Nat Rev Endocrinol. 2011;7:385-95.

10. Alsaleh FM, Smith FJ, Keady S, Taylor KMG. Insulin pumps: from inception to the present and toward the future. J Clin Pharm Ther 2010;35:127-38.

11. Heinemann L. New ways of insulin delivery. Int J Clin Pract. 2011;65:31-46.

12. Murphy HR, Stewart ZA. Automated insulin delivery: what's new, needed, and next? Lancet. 2017;389:333-4.

13. Tauschmann M, Hovorka R. Technology in the management of type 1 diabetes mellitus - current status and future prospects. Nat Rev Endocrinol. 2018;14:464-75.

14. Blauw H, Keith-Hynes P, Koops R, DeVries JH. A review of safety and design requirements of the artificial pancreas. Ann Biomed Eng. 2016;44:3158-72.

15. Ravaine V, Ancla C, Catargi B. Chemically controlled closedloop insulin delivery. J Control Release. 2008;132:2-11.

16. Veiseh O, Tang BC, Whitehead KA, Anderson DG, Langer R. Managing diabetes with nanomedicine: challenges and opportunities. Nat Rev Drug Discov. 2014;14:45-57.

17. VandenBerg MA, Webber MJ. Biologically inspired and chemically derived methods for glucose-responsive insulin therapy. Adv Healthc Mater. 2019;8:1801466.

18. Mo R, Jiang T, Di J, Tai W, Gu Z. Emerging micro- and nanotechnology based synthetic approaches for insulin delivery. Chem Soc Rev. 2014;43:3595-629.

19. Matsumoto A, Miyahara Y. Current development status and perspectives of self-regulated insulin delivery systems: a review. Electron Commun Jpn. 2014;97:57-61.

20. Zhao L, Xiao C, Wang L, Gai G, Ding J. Glucose-sensitive polymer nanoparticles for self-regulated drug delivery. Chem Commun. 2016;52:7633-52.

21. Shen D, Yu H, Wang L, Khan A, Haq F, Chen X, et al. Recent progress in design and preparation of glucose-responsive insulin delivery systems. J Control Release. 2020;321:236-58.

22. Yang J, Cao Z. Glucose-responsive insulin release: analysis of mechanisms, formulations, and evaluation criteria. J Control Release. 2017;263:231-9.

23. Kataoka K, Miyazaki H, Bunya M, Okano T, Sakurai Y. Totally synthetic polymer gels responding to external glucose concentration: their preparation and application to on-off regulation of insulin release. J Am Chem Soc. 1998;120:12694-5.

24. Wang J, Wang Z, Yu J, Kahkoska AR, Buse JB, Gu Z. Glucoseresponsive insulin and delivery systems: innovation and translation. Adv Mater. 2020;32:1902004.

25. Matsumoto A, Kataoka K, Miyahara Y. New directions in the design of phenylboronate-functionalized polymers for diagnostic and therapeutic applications. Polym J. 2014;46:483-91.

26. Matsumoto A, Miyahara Y. 'Borono-lectin' based engineering as a versatile platform for biomedical applications. Sci Technol Adv Mater. 2018;19:18-30.

27. Zhao L, Huang Q, Liu Y, Wang Q, Wang L, Xiao S, et al. Boronic acid as glucose-sensitive agent regulates drug delivery for diabetes treatment. Materials. 2017;10:170.

28. Lor JP, Edwards JO. Polyol complexes and structure of the benzeneboronate ion. J Org Chem. 1959;24:769-74.

29. Aronoff S, Chen TC, Cheveldayoff M. Complexation of Dglucose with borate. Carbohydr Res. 1975;40:299-309.

30. Kitano S, Koyama Y, Kataoka K, Okano T, Sakurai Y. A novel drug delivery system utilizing a glucose responsive polymer complex between poly (vinyl alcohol) and poly (N-vinyl-2- pyrrolidone) with a phenylboronic acid moiety. J Control Release. 1992;19:161-70.

31. Kitano S, Kataoka K, Koyama Y, Okano T, Sakurai Y. Glucoseresponsive complex formation between poly(vinyl alcohol) and poly(N-vinyl-2-pyrrolidone) with pendent phenylboronic acid moieties. Die Makromol Chemie Rapid Commun. 1991;12: 227-33.

32. Martin AR, Vasseur J-J, Smietana M. Boron and nucleic acid chemistries: merging the best of both worlds. Chem Soc Rev. 2013;42:5684

33. Matsumoto A, Ikeda S, Atsushi Harada A, Kataoka K. Glucoseresponsive polymer bearing a novel phenylborate derivative as a glucose-sensing moiety operating at physiological $\mathrm{pH}$ conditions. Biomacromolecules. 2003;4:1410-6.

34. Sharifzadeh G, Hosseinkhani H. Biomolecule-responsive hydrogels in medicine. Adv Healthc Mater 2017;6:1700801.

35. Ma R, Shi L. Phenylboronic acid-based glucose-responsive polymeric nanoparticles: synthesis and applications in drug delivery. Polym Chem. 2014;5:1503-18.

36. Shiino D, Murata Y, Kubo A, Kim YJ, Kataoka K, Koyama Y, et al. Amine containing phenylboronic acid gel for glucoseresponsive insulin release under physiological $\mathrm{pH}$. J Control Release. 1995;37:269-76.

37. Deng CC, Brooks WLA, Abboud KA, Sumerlin BS. Boronic acid-based hydrogels undergo self-healing at neutral and acidic pH. ACS Macro Lett. 2015;4:220-4.

38. Matsumoto A, Ryo Yoshida A, Kataoka K. Glucose-responsive polymer gel bearing phenylborate derivative as a glucose-sensing moiety operating at the physiological $\mathrm{pH}$. Biomacromolecules. 2004;5:1038-45.

39. Matsumoto A, Yamamoto K, Yoshida R, Kataoka K, Aoyagi T, Miyahara Y. A totally synthetic glucose responsive gel operating in physiological aqueous conditions. Chem Commun. 2010;46: 2203-5.

40. Matsumoto A, Ishii T, Nishida J, Matsumoto H, Kataoka K, Miyahara Y. A synthetic approach toward a self-regulated insulin delivery system. Angew Chem Int Ed. 2012;51:2124-8.

41. Washburn RM, Billig FA, BLOOM M, Albright CF, Levens E. Organoboron Compounds in Borax to boranes, Advances in chemistry, 32. Washington: ACS Publication; 1961, Chapter 23, 208-20.

42. Matsumoto A, Yuasa M, Matsumoto H, Sanjo M, Tabata M, Goda $\mathrm{T}$, et al. Boronate-functionalized polymer gel-based insulin delivery system with improved stability in performance: a comparative structure-function study. Chem Lett. 2016;45:460-2.

43. Matsumoto A, Kurata T, Shiino D, Kataoka K. Swelling and shrinking kinetics of totally synthetic, glucose-responsive polymer gel bearing phenylborate derivative as a glucose-sensing moiety. Macromolecules. 2004;37:1502-10.

44. Yu J, Zhang Y, Bomba H, Gu Z. Stimuli-responsive delivery of therapeutics for diabetes treatment. Bioeng Transl Med. 2016;1:323-37.

45. Matsumoto A, Tanaka M, Matsumoto H, Ochi K, Moro-oka Y, Kuwata H, et al. Synthetic "smart gel" provides glucose-responsive insulin delivery in diabetic mice. Sci Adv. 2017;3:eaaq0723.

46. Matsumoto A, Kuwata H, Kimura S, Matsumoto H, Ochi K, Moro-oka Y, et al. Hollow fiber-combined glucose-responsive gel technology as an in vivo electronics-free insulin delivery system. Commun Biol. 2020;3:313.

47. Halder J, Gupta S, Kumari R, Gupta GD, Rai VK. Microneedle array: applications, recent advances, and clinical pertinence in transdermal drug delivery. J Pharm Innov. 2020;8:1-8.

48. Jin X, Zhu DD, Chen BZ, Ashfaq M, Guo XD. Insulin delivery systems combined with microneedle technology. Adv Drug Deliv Rev. 2018;127:119-37. 
49. Hu Q, Chen $\mathrm{Q}, \mathrm{Gu} \mathrm{Z}$. Advances in transformable drug delivery systems. Biomaterials. 2018;178:546-58.

50. Dardano P, Battisti M, Rea I, Serpico L, Terracciano M, Cammarano A, et al. Polymeric microneedle arrays: versatile tools for an innovative approach to drug administration. Adv Ther. 2019;2:1900036.

51. Yang R, Wei T, Goldberg H, Wang W, Cullion K, Kohane DS. Getting drugs across biological barriers. Adv Mater. 2017;29: 1606596.

52. Singh P, Carrier A, Chen Y, Lin S, Wang J, Cui S, et al. Polymeric microneedles for controlled transdermal drug delivery. $\mathrm{J}$ Control Release. 2019;315:97-113.

53. Liu GS, Kong Y, Wang Y, Luo Y, Fan X, Xie X, et al. Microneedles for transdermal diagnostics: recent advances and new horizons. Biomaterials. 2020;232:119740.

54. Ronnander JP, Simon L, Koch A. Transdermal delivery of sumatriptan succinate using iontophoresis and dissolving microneedles. J Pharm Sci. 2019;108:3649-56.
55. Chen X, Wang L, Yu H, Li C, Feng J, Haq F, et al. Preparation, properties and challenges of the microneedles-based insulin delivery system. J Control Release. 2018;288:173-88.

56. Chen G, Yu J, Gu Z. Glucose-responsive microneedle patches for diabetes treatment. J Diabetes Sci Technol. 2019;13:41-48.

57. Yu J, Zhang Y, Kahkoska AR, Gu Z. Bioresponsive transcutaneous patches. Curr Opin Biotechnol. 2017;48:28-32.

58. Chen S, Matsumoto H, Moro-oka Y, Tanaka M, Miyahara Y, Suganami T, et al. Microneedle-array patch fabricated with enzyme-free polymeric components capable of on-demand insulin delivery. Adv Funct Mater. 2019;29:1807369.

59. Chen S, Matsumoto H, Moro-Oka Y, Tanaka M, Miyahara Y, Suganami T, et al. Smart microneedle fabricated with silk fibroin combined semi-interpenetrating network hydrogel for glucoseresponsive insulin delivery. ACS Biomater Sci Eng. 2019;5:5781-9.

60. Chen S, Miyazaki T, Itoh M, Matsumoto H, Morooka Y, Tanaka $\mathrm{M}$, et al. Temperature-stable boronate gel based microneedle technology for self-regulated insulin delivery. ACS Appl Polym Mater. 2020;2:2781-90.

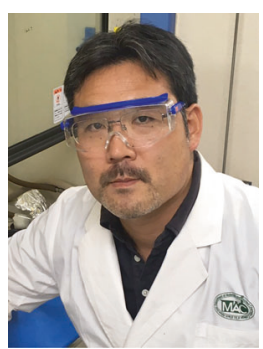

Akira Matsumoto is a Research Professor in Institute of Biomaterials and Bioengineering at Tokyo Medical and Dental University (TMDU), and is a Project Leader at Kanagawa Institute of Industrial Science and Technology (KISTEC). He serves a vice president of SPSJ Research Group on Polymer Gels, and an Editorial Board Member of Gels. After getting his Ph.D. from the University of Tokyo (UT) in 2004, he joined a group of Prof. David L. Kaplan at Tufts University as a Postdoctoral Fellow, returned in 2006 to UT as an Assistant Professor, and moved to TMDT in 2010. Among other honors he has been awarded SPSJ Asahi Kasei Award 2020, Bioindustry Research Award 2018, TMDU Award for Excellence in Research in 2013, and the Nakatani Award in 2012. With a background on polymer materials chemistry, he has been fascinated by boronic acid-based molecular-recognition chemistry, termed "borono-lectins," as a versatile platform for biomedical applications, including insulin delivery systems and cancer diagnosis and therapy.

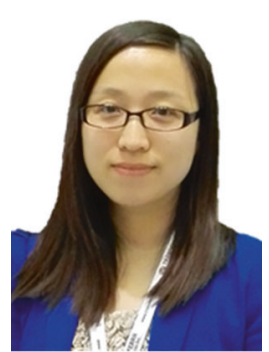

Siyuan Chen received her B.Sc. and M.Sc. degree from Huazhong University of Science and Technology and obtained her Ph.D. degree from Imperial College London in 2017. She worked as a researcher at Kanagawa Institute of Industrial Science and Technology from 2017 to 2020. In 2021, she joined the Department of Material Science and Technology at Nanjing Tech University as a professor. Her research mainly focuses on the development of stimuli-responsive biomaterials and delivery systems for smart drug delivery, including phenylboronic acid gel-combined microneedle for glucose-responsive transdermal insulin delivery, virus-mimicking multifunctional liposomes for cancer therapy, and DMSO-free cell cryopreservation. 